\title{
Early Use of TIPS in Patients with Cirrhosis and Variceal Bleeding
}

\author{
Juan Carlos García-Pagán, M.D., Karel Caca, M.D., Christophe Bureau, M.D., \\ Wim Laleman, M.D., Beate Appenrodt, M.D., Angelo Luca, M.D., \\ Juan G. Abraldes, M.D., Frederik Nevens, M.D., Jean Pierre Vinel, M.D., \\ Joachim Mössner, M.D., and Jaime Bosch, M.D., for the Early TIPS \\ (Transjugular Intrahepatic Portosystemic Shunt) Cooperative Study Group
}

ABSTRACT

From the Hepatic Hemodynamic Laboratory, Liver Unit, Institut de Malalties Digestives i Metaboliques, University of Barcelona, and Hospital Clínic, Institut d'Investigacions Biomèdiques August $\mathrm{Pi}$ i Sunyer; and Centro de Investigación Biomédica en Red de Enfermedades Hepáticas y Digestivas (J.C.G.-P., J.G.A., J.B.) - all in Barcelona; Medizinische Klinik I, Klinikum Ludwigsburg, Ludwigsburg (K.C.); Medical Department I, University of Bonn, Bonn (B.A.); and Medizinische Klinik and Poliklinik I, Universitätsklinikum Leipzig, Leipzig (J.M.) - all in Germany; Service d'Hépatogastro-entérologie, Centre Hospitalier Universitaire de Purpan, Toulouse, France (C.B., J.P.V.); the Department of Hepatology, University Hospital Gasthuisberg, Katholieke Universiteit Leuven, Leuven, Belgium (W.L., F.N.); and Istituto Mediterraneo per i Trapianti e Terapie ad Alta Specializzazione, Palermo, Italy (A.L.). Address reprint requests to Dr. GarcíaPagán or Dr. Bosch at the Hepatic Hemodynamic Laboratory, Liver Unit, Hospital Clínic, Villarroel 170, Barcelona 08036, Spain, or at jcgarcia@clinic.ub.es or jbosch@clinic.ub.es.

N EnglJ Med 2010;362:2370-9.

Copyright ( 2010 Massachusetts Medical Society.

\section{BACKGROUND}

Patients with cirrhosis in Child-Pugh class C or those in class B who have persistent bleeding at endoscopy are at high risk for treatment failure and a poor prognosis, even if they have undergone rescue treatment with a transjugular intrahepatic portosystemic shunt (TIPS). This study evaluated the earlier use of TIPS in such patients.

\section{METHODS}

We randomly assigned, within 24 hours after admission, a total of 63 patients with cirrhosis and acute variceal bleeding who had been treated with vasoactive drugs plus endoscopic therapy to treatment with a polytetrafluoroethylene-covered stent within 72 hours after randomization (early-TIPS group, 32 patients) or continuation of vasoactive-drug therapy, followed after 3 to 5 days by treatment with propranolol or nadolol and long-term endoscopic band ligation (EBL), with insertion of a TIPS if needed as rescue therapy (pharmacotherapy-EBL group, 31 patients).

\section{RESULTS}

During a median follow-up of 16 months, rebleeding or failure to control bleeding occurred in 14 patients in the pharmacotherapy-EBL group as compared with 1 patient in the early-TIPS group $(\mathrm{P}=0.001)$. The 1 -year actuarial probability of remaining free of this composite end point was $50 \%$ in the pharmacotherapy-EBL group versus $97 \%$ in the early-TIPS group $(\mathrm{P}<0.001)$. Sixteen patients died $(12$ in the pharmacotherapy-EBL group and 4 in the early-TIPS group, $\mathrm{P}=0.01$ ). The 1 -year actuarial survival was $61 \%$ in the pharmacotherapy-EBL group versus $86 \%$ in the earlyTIPS group $(\mathrm{P}<0.001)$. Seven patients in the pharmacotherapy-EBL group received TIPS as rescue therapy, but four died. The number of days in the intensive care unit and the percentage of time in the hospital during follow-up were significantly higher in the pharmacotherapy-EBL group than in the early-TIPS group. No significant differences were observed between the two treatment groups with respect to serious adverse events.

\section{CONCLUSIONS}

In these patients with cirrhosis who were hospitalized for acute variceal bleeding and at high risk for treatment failure, the early use of TIPS was associated with significant reductions in treatment failure and in mortality. (Current Controlled Trials number, ISRCTN58150114.) 
V ARICEAL BLEEDING IS A SEVERE COMPLIcation of portal hypertension and a major cause of death in patients with cirrhosis. Advanced liver failure, failure to control variceal bleeding, early rebleeding, and marked elevations in portal pressure are associated with increased mortality. ${ }^{1-3}$ Combined treatment with vasoactive drugs, prophylactic antibiotics, and endoscopic techniques is the recommended standard of care for patients with acute variceal bleeding. ${ }^{4,5}$ However, treatment failure occurs in about 10 to $15 \%$ of patients, ${ }^{6,7}$ who require repeat endoscopic treatments and multiple transfusions. ${ }^{8-10}$ Treatment with a transjugular intrahepatic portosystemic shunt (TIPS) is highly effective in the control of bleeding in such patients, but mortality is still very high, ${ }^{9}$ probably because of further deterioration due to liver failure.

In a study involving patients at high risk for treatment failure, as indicated by a hepatic venous pressure gradient of $20 \mathrm{~mm} \mathrm{Hg}$ or more, ${ }^{11}$ early treatment with TIPS improved the prognosis in comparison with medical treatment in a study by Monescillo et al. ${ }^{12}$ However, the treatment administered in the medical-treatment group was not the current standard of care, ${ }^{12}$ which may have resulted in a worse outcome than expected in this group.

We conducted a study to determine whether early treatment with TIPS, with the use of a stent covered with extended polytetrafluoroethylene (e-PTFE), can improve outcomes in patients with cirrhosis and variceal bleeding who are at high risk for treatment failure and death.

\section{METHODS}

\section{SELECTION OF PATIENTS}

We enrolled patients at nine European centers (see the Supplementary Appendix, available with the full text of this article at NEJM.org) between May 2004 and March 2007. Eligible patients had cirrhosis with acute esophageal variceal bleeding that was being treated with a combination of vasoactive drugs, endoscopic treatment, and prophylactic antibiotics. Patients had Child-Pugh class $C$ disease (a score of 10 to 13) or they had class B disease (a score of 7 to 9) but with active bleeding at diagnostic endoscopy. Patients with scores higher than 13 were excluded from the study. (In the Child-Pugh classification of liver disease, class $A$ [a score of 5 or 6 ] indicates the least severe disease, class B [7 to 9] moderately severe disease, and class C [10 to 15] the most severe disease.) Endoscopic band ligation (EBL) or endoscopic injection sclerotherapy (EIS) was applied at the time of diagnostic endoscopy, performed within 12 hours after admission, while the patients were already receiving vasoactive drugs (terlipressin [2 mg every 4 hours], somatostatin [250 to $500 \mu \mathrm{g}$ per hour], or octreotide [50 to 100 $\mu \mathrm{g}$ per hour]). Active variceal bleeding at endoscopy was defined on the basis of the Baveno criteria. ${ }^{13}$

Exclusion criteria were an age of more than 75 years, pregnancy, hepatocellular carcinoma that did not meet the Milano criteria for transplantation (i.e., a single lesion $<5 \mathrm{~cm}$ or multiple lesions [maximum of three], the largest of which measures $\leq 3 \mathrm{~cm}$ ), a creatinine level greater than $3 \mathrm{mg}$ per deciliter (265 $\mu \mathrm{mol}$ per liter), a Child-Pugh score above 13 points, previous pharmacologic therapy combined with endoscopic treatment to prevent rebleeding, previous use of a portosystemic shunt or TIPS, bleeding from isolated gastric or ectopic varices, total portal-vein thrombosis, and heart failure.

All patients provided written informed consent. The study protocol was approved by the ethics committees of all participating hospitals and followed the Guidelines for Good Clinical Practice in clinical trials.

\section{STUDY DESIGN}

Randomization was performed within 24 hours after admission. The randomization sequence was generated by computer with the use of a concealed block size of four. The coded treatment assignments were kept at the coordinating center in sealed, consecutively numbered, opaque envelopes. Randomized assignments to the study groups were made by contacting the coordinating center (available 24 hours a day) by telephone or fax.

\section{DRUG THERAPY PLUS EBL}

Treatment with vasoactive drugs was continued until patients were free of bleeding for at least 24 hours and preferably up to 5 days, at which point treatment with a nonselective beta-blocker (either propranolol or nadolol) was started. The dose was increased in a stepwise fashion every 2 to 3 days to the maximum tolerated dose or to a maximum of $160 \mathrm{mg}$ twice daily for propranolol and $240 \mathrm{mg}$ per day for nadolol. After these doses were achieved, $10 \mathrm{mg}$ of isosorbide-5-mononitrate was initiated at bedtime, with a stepwise increase in the dose to a maximum of $20 \mathrm{mg}$ twice a day or 
the maximum tolerated dose. In addition, within 7 to 14 days after the initial endoscopic treatment, the second, elective session of EBL was performed. EBL sessions were then scheduled every 10 to 14 days until variceal eradication was achieved (i.e., varices disappeared or could not be suctioned into the banding device).

EBL sessions were performed with the use of multiband devices (6-Shooter Saeed Multi-Band Ligator, Cook, or Speedband SuperView Super 7, Boston Scientific), and bands were applied starting at the gastroesophageal junction. Patients received proton-pump inhibitors until variceal eradication was accomplished. After eradication, endoscopic monitoring was performed at 1-month, 6-month, and 12-month intervals and then annually. If varices reappeared, further EBL sessions were initiated.

Treatment failure was defined as one severe rebleeding episode (i.e., requiring a transfusion of more than 2 units of blood) or two, less severe rebleeding episodes ${ }^{13}$ with TIPS and placement of an e-PTFE-covered stent used as rescue therapy when necessary.

\section{EARLY TIPS}

TIPS was performed within 72 hours after diagnostic endoscopy (or, when possible, within the first 24 hours), and vasoactive drugs were administered until then. The e-PTFE-covered stents (we purchased Viatorr TIPS endoprosthesis, Gore) were initially dilated to $8 \mathrm{~mm}$. If the portal-pressure gradient (the difference between portal-vein pressure and inferior vena caval pressure) did not decrease to below $12 \mathrm{~mm} \mathrm{Hg}$, the stent was dilated to $10 \mathrm{~mm}$.

TIPS revision was performed if there was clinical recurrence of portal hypertension or evidence of TIPS dysfunction on Doppler ultrasonography (i.e., portal blood-flow velocity of $<28 \mathrm{~cm}$ per second, a change in the direction of flow in intrahepatic portal branches, or a drop in portal bloodflow velocity of $>50 \%){ }^{14}$ If TIPS dysfunction was confirmed, angioplasty was performed or another e-PTFE-covered stent was placed.

\section{FOLLOW-UP}

Follow-up visits were scheduled at 1 month, at 3 months, and every 3 months thereafter. Doppler ultrasonography was performed at the first visit, at 6 months, and every 6 months thereafter. Patients were followed until death or liver trans- plantation up to a maximum of 2 years of follow-up or until the end of the study (September 2007).

\section{STUDY END POINTS}

The primary end point of the study was a composite outcome of failure to control acute bleeding or failure to prevent clinically significant variceal rebleeding within 1 year after enrollment. Secondary end points were mortality at 6 weeks and at 1 year, failure to control acute bleeding, early rebleeding (rate of rebleeding at 5 days and at 6 weeks), rate of rebleeding between 6 weeks and 1 year, the development of other complications related to portal hypertension on follow-up, the number of days in the intensive care unit, the percentage of follow-up days spent in the hospital, and the use of alternative treatments.

\section{STATISTICAL ANALYSIS}

In a study by Villanueva et al. involving patients with active variceal bleeding at endoscopy, the rate of failure to control bleeding within 5 days, despite the combined use of drugs plus endoscopic treatment, was $27 \% .{ }^{15}$ Similarly, the rate of failure was $23 \%$ in a study involving patients with Child-Pugh class B or C disease who received combined pharmacologic and endoscopic treatment. ${ }^{16}$ We assumed in our population a $25 \%$ rate of failure to control bleeding at 5 days. Considering that rebleeding occurred between day 5 and 1 year in an additional $20 \%, 6,17$ the cumulative rate of failure to control bleeding or rebleeding at 1 year was estimated to be $45 \%$. We hypothesized that this risk would be reduced to $10 \%$ by an early decision to use e-PTFE-covered TIPS. Because the only rationale for early use of TIPS would be evidence that this approach is better than the current standard treatment, the sample size was calculated with the use of a one-sided test. ${ }^{18,19}$ We calculated that 31 patients per group would be needed to detect these differences, with an alpha level of 0.05 and a beta level of 0.20 .

All data analyses were performed on an intention-to-treat basis according to a preestablished analysis plan. Dichotomous variables were compared by means of Fisher's exact test, and continuous variables were compared by means of the nonparametric Mann-Whitney rank-sum test. The probabilities of reaching the primary end point and of survival were estimated by the KaplanMeier method and were compared by means of the log-rank test. Absolute risk reductions and 


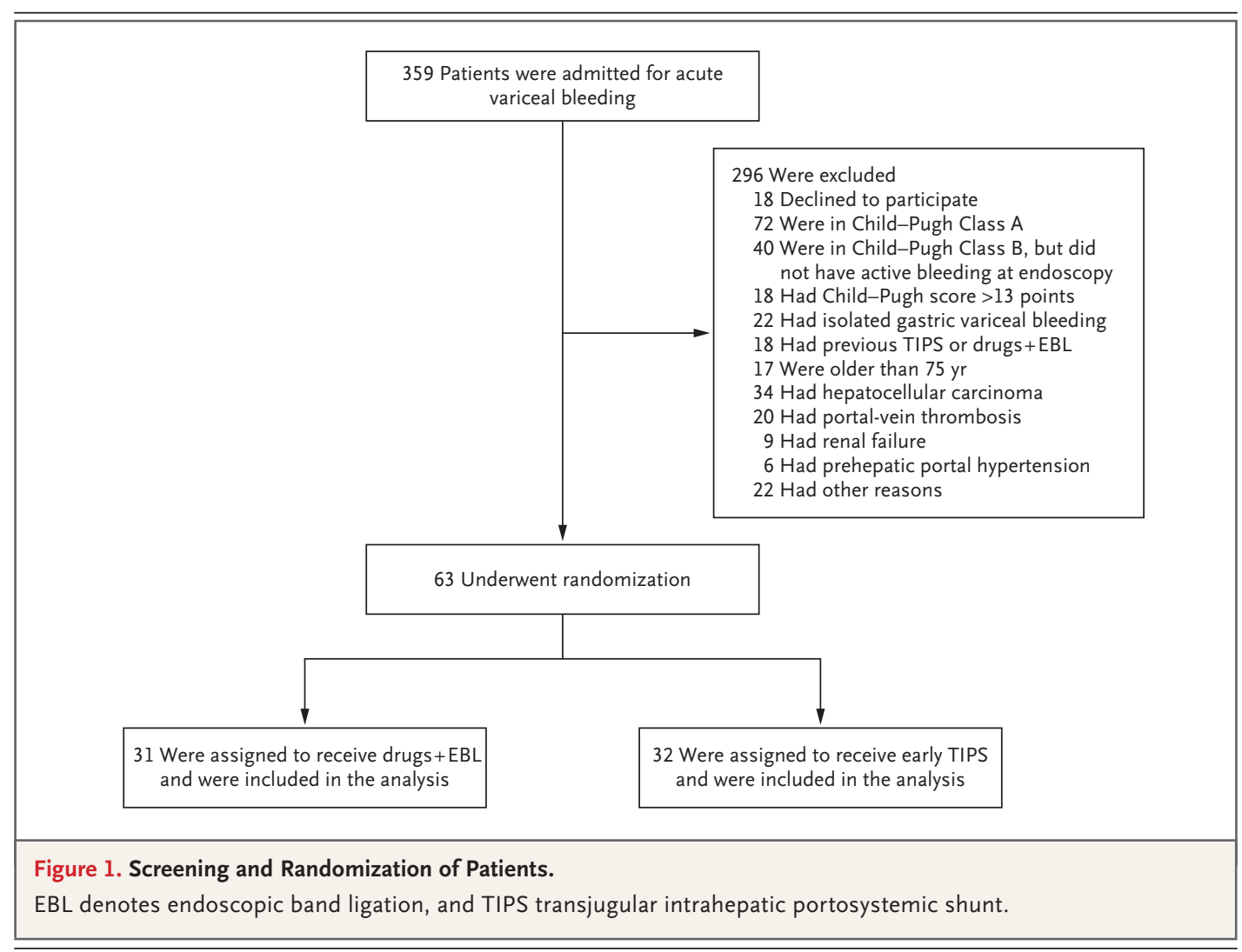

number needed to treat, with $95 \%$ confidence intervals, were calculated as an estimate of the effect size and its precision. A P value of less than 0.05 was considered to indicate statistical significance, and all tests were two-sided. The statistical software packages used for the analysis were SPSS (version 16.0) and confidence interval analysis (CIA, version 2.1.2, University of Southampton, United Kingdom).

\section{RESULTS}

\section{STUDY PATIENTS}

We screened 359 patients with acute variceal bleeding who were admitted to the participating hospitals for study eligibility. A total of 296 patients were excluded (Fig. 1), and the remaining 63 patients were randomly assigned to either the pharmacotherapy-EBL group (31 patients) or the early-TIPS group (32 patients). There were no significant differences in baseline characteristics between the two groups at the time of entry into the study (Table 1). A total of 7 patients ( 3 in the pharmacotherapy-EBL group and 4 in the earlyTIPS group) were lost to follow-up after a median of 8 months (range, 0.5 to 12) without reaching the composite end point. The mean $( \pm \mathrm{SD})$ followup period was 10.6 \pm 9.9 months in the pharmacotherapy-EBL group and 14.6 \pm 8.4 months in the early-TIPS group. A total of 6 patients ( 2 in the pharmacotherapy-EBL group and 4 in the earlyTIPS group) underwent liver transplantation during follow-up.

In the pharmacotherapy-EBL group, 22 patients received propranolol (median dose, $55 \mathrm{mg}$ [range, 10 to 160]), and only 3 received nadolol. In the remaining 6 patients, nonselective beta-blocker therapy was not initiated because of failure to control bleeding, early rebleeding, or death. In 12 patients, isosorbide-5-mononitrate was added to the nonselective beta-blocker (median dose, $25 \mathrm{mg}$ [range, 10 to 40]), but it was not added in 13 patients because of arterial hypotension, the treating physician's preference, or early death. Variceal eradication was achieved in 12 patients after a median of 2 EBL sessions (range, 1 to 7) without rebleeding; in 4 patients, eradication was achieved after treatment of a rebleeding episode with additional EBL sessions. In the remaining 15 patients, eradication was not achieved (in 12 because 
Table 1. Baseline Characteristics of the Patients.*

\section{Characteristic}

Age (yr)

Sex (no. of patients)

Male

Female

Cause of cirrhosis (no. of patients)

Alcohol

$\mathrm{HCV}$

Other

Active alcoholism (no. of patients)

Child-Pugh classification (no. of patients) $\dagger$

Class B

Class C

Child-Pugh score

MELD scoreł

MELD-Na score $\mathbb{8}$

Ascites (no. of patients)

Bilirubin ( $\mathrm{mg} / \mathrm{dl})$

Albumin (g/liter)

Prothrombin time (\%) 9

Creatinine $(\mathrm{mg} / \mathrm{dl})$

Previous hepatic encephalopathy (no. of patients)

Previous variceal bleeding in the absence of combined treatment (no. of patients)

Active bleeding at endoscopy (no. of patients)

Shock at time of admission (no. of patients)

Systolic blood pressure $(\mathrm{mm} \mathrm{Hg})$

Hematocrit on admission (\%)

Units of blood transfused before randomization (no.)

Endoscopic treatment at time of index bleeding (no. of patients)

Band ligation

Injection sclerotherapy

Vasoactive-drug therapy at time of index bleeding (no. of patients)

Terlipressin

Somatostatin

Octreotide

\section{Pharmacotherapy-EBL Early-TIPS Group Group ( $N=31)$ \\ $(\mathrm{N}=32)$}

$49 \pm 6$

P Value

0.22

0.78

23

21

11

$20 \quad 22$

5

6

17

0.61

0.99

$16 \quad 16$

$15 \quad 16$

$9.5 \pm 1.8$

$9.3 \pm 1.8$

0.66

$16.9 \pm 6.3$

$15.5 \pm 5$

0.28

$19 \pm 7$

$17 \pm 6$

0.25

18

19

0.99

$4.4 \pm 4.9$

$3.7 \pm 4.8$

0.34

$26 \pm 7$

$26 \pm 7$

0.89

$50 \pm 15$

$53 \pm 15$

0.33

$1 \pm 0.4$

0

$1 \pm 0.5$

0.59

6

0.03

5

11

0.15

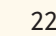

7

$111 \pm 23$

$27 \pm 8$

$2.9 \pm 3$

23

0.99

0.99

0.22

0.49

0.73

0.17

26

5
21

11

0.74

* Plus-minus values are means \pm SD. To convert the values for bilirubin to micromoles per liter, multiply by 17.1. To convert the values for creatinine to micromoles per liter, multiply by 88.4. HCV denotes hepatitis C virus, and TIPS transjugular intrahepatic portosystemic shunt.

$\uparrow$ According to the Child-Pugh classification of liver disease, class A (5 to 6 points) indicates the least severe disease, class B ( 7 to 9 points) moderately severe disease, and class C (10 to 15 points) the most severe disease. Patients whose Child-Pugh score exceeded 13 points were not included in the study.

$¥$ The Model for End-Stage Liver Disease (MELD) score ranges from 6 to 40, with higher scores indicating more severe disease.

$\int$ The MELD-Na score ranges from 6 to 40, with higher scores indicating more severe liver disease.

q The values shown represent a proportion of the mean prothrombin time in a group of control subjects.

The New England Journal of Medicine 


\begin{tabular}{|c|c|c|c|}
\hline Variable & $\begin{array}{l}\text { Pharmacotherapy-EBL } \\
\text { Group }(\mathrm{N}=31)\end{array}$ & $\begin{array}{l}\text { Early-TIPS Group } \\
\quad(\mathrm{N}=32)\end{array}$ & $P$ Value \\
\hline Composite end point reached (no. of patients) & 14 & 1 & 0.001 \\
\hline \multicolumn{4}{|l|}{ Child-Pugh classification †' } \\
\hline Class B & 5 & 0 & \\
\hline Class C & 9 & 1 & \\
\hline Treatment failure at 5 daystr & 4 & 1 & \\
\hline \multicolumn{4}{|l|}{ Rebleeding } \\
\hline$>5$ days -6 wk & 7 & 0 & \\
\hline$>6 \mathrm{wk}-1 \mathrm{yr}$ & 3 & 0 & \\
\hline Minor rebleeding (no. of patients) & 3 & 1 & 0.35 \\
\hline Gastric ulcer & 1 & & \\
\hline Portal hypertensive gastropathy & 1 & & \\
\hline Varices & 1 & & \\
\hline Post-EBL ulcer & & 1 & \\
\hline Days in intensive care unit (no.) & $8.6 \pm 9$ & $3.6 \pm 4$ & 0.01 \\
\hline Orthotopic liver transplantation (no. of patients) & 2 & 4 & 0.67 \\
\hline Death (no. of patients) & 12 & 4 & 0.01 \\
\hline \multicolumn{4}{|l|}{ Child-Pugh classification } \\
\hline Class B & 2 & 1 & \\
\hline Class C & 10 & 3 & \\
\hline \multicolumn{4}{|l|}{ Cause of death } \\
\hline Recurrent bleeding & 5 & 0 & \\
\hline Sepsis & 4 & 3 & \\
\hline Liver failure & 2 & 0 & \\
\hline Hepatorenal syndrome & 1 & 0 & \\
\hline Arrhythmia & 0 & 1 & \\
\hline Time in hospital (\% of follow-up) & & & 0.014 \\
\hline Median & 15 & 4 & \\
\hline Interquartile range & $5-100$ & $2-13$ & \\
\hline
\end{tabular}

* Plus-minus values are means \pm SD. EBL denotes endoscopic band ligation, and TIPS transjugular intrahepatic portosystemic shunt.

$\uparrow$ According to the Child-Pugh classification of liver disease, class A (5 to 6 points) indicates the least severe disease, class B ( 7 to 9 points) moderately severe disease, and class C (10 to 15 points) the most severe disease. Patients whose Child-Pugh score exceeded 13 points were not included in the study.

$\uparrow$ Treatment failure was defined as one severe rebleeding episode (with transfusion requiring more than 2 units of blood) or two less severe rebleeding episodes.

the primary end point was reached [resulting in rescue TIPS in 7 and death in 5], in 2 who were lost to follow-up, and in 1 despite eight EBL sessions).

In the early-TIPS group, all but 1 patient, who withdrew consent, underwent early shunt placement. There were no technical failures or major complications of the TIPS procedure. Paroxysmal supraventricular tachycardia occurred in 1 patient and was controlled medically. A total of 27 patients required one stent, and 4 required two stents. The mean portal-pressure gradient dropped from $20.2 \pm 7 \mathrm{~mm} \mathrm{Hg}$ to $6.2 \pm 3 \mathrm{~mm} \mathrm{Hg}(\mathrm{P}<0.001)$. Despite dilation to $10 \mathrm{~mm}$, the portal-pressure gradient after TIPS remained above $12 \mathrm{~mm} \mathrm{Hg}$ in 2 patients. Collateral embolization was performed in 2 patients (one of whom had a portal-pressure gradient above $12 \mathrm{~mm} \mathrm{Hg}$ after TIPS). 


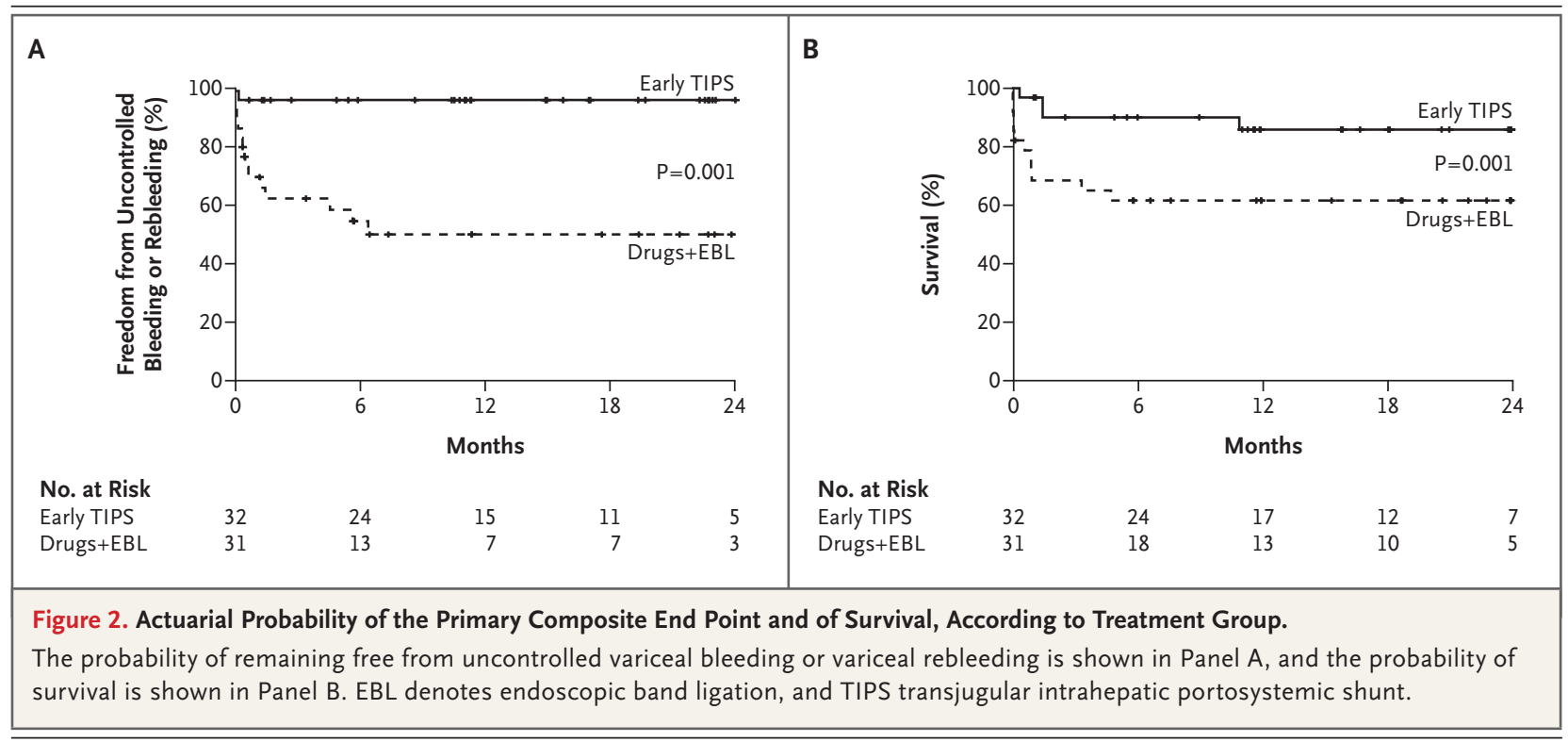

\section{REBLEEDING}

In the pharmacotherapy-EBL group, 14 patients reached the primary composite end point (Table 2 and Fig. 2). In these patients, the Model for EndStage Liver Disease (MELD) score (which ranges from 6 to 40, with higher scores indicating more severe disease) increased from a mean of $18.8 \pm 6.4$ at randomization to $22.6 \pm 11$ at the time the end point was reached. In 7 of these patients, TIPS with an e-PTFE-covered stent was used as rescue therapy; although bleeding was controlled, 4 of these patients died within 36 days (range, 1 to 36). In 5 patients, no further treatment was considered because of severe liver failure, and all died. In the 9 patients who died, the mean MELD score was $28.2 \pm 9$ when they reached the primary end point of the study. The remaining 2 patients who reached the primary end point underwent additional EBL sessions and were alive at the end of the follow-up period.

Only 1 patient in the early-TIPS group, in whom the portal-pressure gradient remained above 12 $\mathrm{mm} \mathrm{Hg}$, reached the primary end point $(\mathrm{P}<0.001$ for the comparison with the pharmacotherapyEBL group) (Table 2). The 1-year actuarial probability of remaining free of failure to control bleeding and of variceal rebleeding was significantly higher in the early-TIPS group than in the pharmacotherapy-EBL group ( $97 \%$ vs. $50 \%$; absolute risk reduction, 47 percentage points; $95 \%$ confidence interval [CI], 25 to 69; number needed to treat, 2.1 patients; 95\% CI, 1.4 to 4.0) (Fig. 2). An additional 4 patients ( 3 in the pharmacotherapyEBL group and 1 in the early-TIPS group) had a rebleeding episode that was not clinically significant (i.e., it did not require hospital admission or blood transfusion) (Table 2).

\section{SURVIVAL}

A total of 12 patients in the pharmacotherapyEBL group died, as compared with 4 in the earlyTIPS group $(\mathrm{P}=0.001)$. The 6 -week survival rate was higher in the early-TIPS group than in the pharmacotherapy-EBL group ( $97 \%$ vs. $67 \%$; absolute risk reduction, 30 percentage points; 95\% CI, 12 to 48 ; number needed to treat, 3.3 patients; $95 \% \mathrm{CI}$, 2.1 to 8.3$)$, as was the 1-year survival rate $(86 \%$ vs. $61 \%$; absolute risk reduction, 25 percentage points; 95\% CI, 2 to 48; number needed to treat, 4.0 patients; 95\% CI, 2.1 to 50.0) (Fig. 2). Causes of death are summarized in Table 2.

\section{OTHER COMPLICATIONS OF PORTAL HYPERTENSION} The 1-year actuarial probability of hepatic encephalopathy was $28 \%$ in the early-TIPS group as compared with $40 \%$ in the pharmacotherapy-EBL group (an absolute difference of 12 percentage points; $95 \% \mathrm{CI},-18$ to 40 ; $\mathrm{P}=0.13$ ). In the pharmacotherapy-EBL group, 12 patients had a total of 17 episodes of hepatic encephalopathy, whereas in the early-TIPS group, 8 patients had a total of 10 episodes (Table 3). Most of these episodes occurred during the index bleeding. After discharge, the 1-year probability of additional epi- 
sodes of hepatic encephalopathy was similar in the two groups ( $10 \%$ in the pharmacotherapy-EBL group and $19 \%$ in the early-TIPS group, $\mathrm{P}=0.80$ ). A total of 3 patients in the pharmacotherapy-EBL group and 2 in the early-TIPS group had stage III hepatic encephalopathy, and 1 patient in each group had mild, recurrent hepatic encephalopathy.

The 1-year actuarial probability of new or worsening ascites was 33\% in the pharmacotherapyEBL group and 13\% in the early-TIPS group - an absolute difference of 20 percentage points ( $95 \%$ $\mathrm{CI},-8$ to $47 ; \mathrm{P}=0.11$ ). Spontaneous bacterial peritonitis developed during the index bleeding in 2 patients in the pharmacotherapy-EBL group, both of whom died. In addition, the hepatorenal syndrome developed during the index bleeding in 7 patients: 5 in the pharmacotherapy-EBL group, 4 of whom died, and 2 in the early-TIPS group, both of whom survived. The proportion of follow-up time that patients spent in the hospital was 15\% (interquartile range, 5 to 100) in the pharmacotherapy-EBL group as compared with $4 \%$ (interquartile range, 2 to 13 ) in the early-TIPS group $(\mathrm{P}=0.01)$.

\section{OTHER ADVERSE EVENTS}

As shown in Table 3, there were no significant between-group differences in the numbers of patients who had adverse effects.

\section{DISCUSSION}

In the study by Monescillo et al., ${ }^{12}$ early treatment with TIPS, as compared with medical treatment, was associated with an improved prognosis among patients at high risk for uncontrolled bleeding or rebleeding on the basis of a hepatic venous pressure gradient of $20 \mathrm{~mm} \mathrm{Hg}$ or higher. ${ }^{11}$ This study did not include continued pharmacologic therapy and EBL in the medical-treatment group, and bare stents were used in the early-TIPS group. ${ }^{12}$ The use of e-PTFE-covered stents is associated with reductions in the incidence of TIPS dysfunction and recurrence of complications related to portal hypertension. ${ }^{20,21}$ More important, the decision to perform TIPS was based on the measurement of hepatic venous pressure gradients, a test that is not widely available, especially under emergency conditions. Therefore, it is difficult to extrapolate the results of this study for application to clinical practice.

Our study was specifically designed to show
Table 3. Adverse Events.*

\begin{tabular}{|c|c|c|}
\hline Adverse Event & $\begin{array}{l}\text { Pharmacotherapy- } \\
\text { EBL Group }(N=31)\end{array}$ & $\begin{array}{c}\text { Early-TIPS } \\
\text { Group }(\mathrm{N}=32)\end{array}$ \\
\hline & \multicolumn{2}{|c|}{ no. of patients } \\
\hline \multicolumn{3}{|l|}{ Complications of portal hypertension } \\
\hline Hepatic encephalopathy & 12 & 8 \\
\hline Ascites & 9 & 5 \\
\hline Spontaneous bacterial peritonitis & 2 & 0 \\
\hline Hepatorenal syndrome & 5 & 2 \\
\hline \multicolumn{3}{|l|}{ Other serious adverse events } \\
\hline $\begin{array}{l}\text { Infections (other than pneumonia or } \\
\text { spontaneous bacterial peritonitis) }\end{array}$ & 4 & 4 \\
\hline Pneumonia & 3 & 3 \\
\hline Acute episode in chronic liver failure & 1 & 3 \\
\hline Delirium tremens & 2 & 1 \\
\hline Korsakoff syndrome & 0 & 1 \\
\hline Esophageal perforation & 1 & 0 \\
\hline Dysphagia & 1 & 0 \\
\hline Bleeding from banding-related ulcers & 1 & 0 \\
\hline Choledocholithiasis & 0 & 1 \\
\hline Alcoholic hepatitis & 0 & 1 \\
\hline Bleeding duodenal ulcer & 0 & 1 \\
\hline \multicolumn{3}{|l|}{ Nonserious adverse events } \\
\hline Gynecomastia & 0 & 1 \\
\hline Edema & 1 & 0 \\
\hline Sleep disorder & 1 & 0 \\
\hline Chest pain (after EBL) & 1 & 0 \\
\hline Epistaxis (after EBL) & 1 & 0 \\
\hline Vomiting & 1 & 0 \\
\hline
\end{tabular}

*EBL denotes endoscopic band ligation.

whether an early decision to use TIPS, with e-PTFE-covered stents and based on clinical criteria, can improve the prognosis for patients with variceal bleeding who are at high risk. We found that in patients treated early with TIPS, the risks of failure to control bleeding and of variceal rebleeding were reduced.

In addition, and even more important, the early use of TIPS was associated with a reduction in mortality. This beneficial effect on survival was observed even though rescue TIPS was used in patients in whom medical treatment failed. Mortality was very high among the patients who underwent rescue TIPS after treatment failure, a result that is consistent with the findings in previous 
studies.9,22 Remarkably, in five patients, TIPS was not even considered because of the severe deterioration in liver function, as evidenced by the increased MELD scores, which were higher than those associated with excessive mortality after TIPS. ${ }^{23}$ These data make it clear that for patients in Child-Pugh class C or in class B with active variceal bleeding, failure to initially control the bleeding or early rebleeding contributes to further deterioration in liver function, which in turn worsens the prognosis and may preclude the use of rescue TIPS.

Previous studies evaluating the role of TIPS in the prevention of recurrent variceal bleeding clearly showed that TIPS reduces the rebleeding rate but increases hepatic encephalopathy without improving survival. ${ }^{24-26}$ Because of these findings, TIPS is currently recommended only as a rescue therapy. ${ }^{4,5}$ The results of the present study challenge this recommendation. It should be noted that previous studies of TIPS differed from our study in that they used bare stents or did not limit enrollment to patients at high risk for treatment failure. In the study by Escorsell et al., ${ }^{26}$ more than $15 \%$ of patients with variceal bleeding were excluded because of a need for emergency TIPS or death from uncontrolled bleeding before day 5 . Therefore, the study design precluded the possibility of demonstrating a benefit of TIPS in these high-risk patients. Furthermore, in another study, ${ }^{7}$ mortality was $66 \%$ among patients whose bleeding was not controlled by the initial treatment. In high-risk patients, the potentially deleterious effects of e-PTFE-covered TIPS appear to be counterbalanced by its high efficacy in controlling bleeding and thus preventing further clinical deterioration. In contrast, TIPS should not be used as the initial treatment in patients with Child-
Pugh class A disease, since the rates of medicaltreatment failure and mortality are low among such patients.

Although the risks of treatment failure and death were higher in patients with Child-Pugh class $\mathrm{C}$ disease than in those with class B disease, our trial was not powered to conduct appropriate subgroup analyses. Therefore, further evaluation will be needed to determine whether the early use of TIPS equally benefits these two subgroups of patients.

The early use of TIPS was not associated with an increase in the number or severity of episodes of hepatic encephalopathy.

In conclusion, in patients with Child-Pugh class $\mathrm{C}$ disease or class B disease with active bleeding who were admitted for acute variceal bleeding, the early use of TIPS with an e-PTFE-covered stent was associated with significant reductions in the failure to control bleeding, in rebleeding, and in mortality, with no increase in the risk of hepatic encephalopathy.

Supported in part by grants (FIS 06/0623, 04/1031, and 08/0504) from Instituto de Salud Carlos III (Spain), a grant (SAF07/61298) from Ministerio de Educación y Ciencia (Spain), a grant (04 036 03) from the University Hospital Center of Toulouse, a grant from the Fund for Scientific Research — Flanders (Fundamenteel Klinisch Mandaat - FWO Vlaanderen, to Dr. Laleman), and an educational grant from Gore. Centro de Investigación Biomédica en Red de Enfermedades Hepáticas y Digestivas is funded by Instituto de Salud Carlos III.

Dr. García-Pagán reports receiving grant support from Gore and reimbursement for travel expenses from Gore, Cook Medical, and Ferring; Dr. Bosch, receiving grant support and reimbursement for travel expenses from Gore and consulting fees from Astellas, Actelion, Axcan, NicOx, Chiasma, and Dominion Pharmakine; Dr. Caca, receiving lecture fees and reimbursement for travel expenses from Gore; and Dr. Appenrodt, receiving reimbursement for travel expenses from Gore. No other potential conflict of interest relevant to this article was reported.

Disclosure forms provided by the authors are available with the full text of this article at NEJM.org.
REFERENCES

1. Ben-Ari Z, Cardin F, McCormick AP, Wannamethee G, Burroughs AK. A predictive model for failure to control bleeding during acute variceal haemorrhage. J Hepatol 1999;31:443-50. [Erratum, J Hepatol 2001;34:640.]

2. García-Pagán JC, Teres J, Calvet X, Bosch J, Rodes J. Factors which influence the prognosis of the first episode of variceal hemorrhage in patients with cirrhosis. In: Bosch J, Rodes J, eds. Recent advances in the pathophysiology and therapy of portal hypertension. Barcelona: Ares Serono Symposia, 2004:287-301.

3. Goulis J, Armonis A, Patch D, Sabin
C, Greenslade L, Burroughs AK. Bacterial infection is independently associated with failure to control bleeding in cirrhotic patients with gastrointestinal hemorrhage. Hepatology 1998;27:1207-12.

4. Garcia-Tsao G, Sanyal AJ, Grace ND, Carey WD. Prevention and management of gastroesophageal varices and variceal hemorrhage in cirrhosis. Am J Gastroenterol 2007;102:2086-102.

5. de Franchis R. Evolving consensus in portal hypertension: report of the Baveno IV consensus workshop on methodology of diagnosis and therapy in portal hypertension. J Hepatol 2005;43:167-76.
6. Bosch J, Abraldes JG, Berzigotti A, García-Pagán JC. Portal hypertension and gastrointestinal bleeding. Semin Liver Dis 2008;28:3-25.

7. D'Amico G, de Franchis R. Upper digestive bleeding in cirrhosis: post-therapeutic outcome and prognostic indicators. Hepatology 2003;38:599-612.

8. Bernard B, Cadranel JF, Valla D, Escolano S, Jarlier V, Opolon P. Prognostic significance of bacterial infection in bleeding cirrhotic patients: a prospective study. Gastroenterology 1995;108:1828-34.

9. Azoulay D, Castaing D, Majno P, et al. Salvage transjugular intrahepatic porto- 
systemic shunt for uncontrolled variceal bleeding in patients with decompensated cirrhosis. J Hepatol 2001;35:590-7.

10. Spina GP, Santambrogio R, Opocher E, et al. Emergency portosystemic shunt in patients with variceal bleeding. Surg Gynecol Obstet 1990;171:456-64.

11. Moitinho E, Escorsell A, Bandi JC, et al. Prognostic value of early measurement of portal pressure in acute variceal bleeding. Gastroenterology 1999;117:626-31.

12. Monescillo A, Martinez-Lagares F, Ruiz-del-Arbol L, et al. Influence of portal hypertension and its early decompression by TIPS placement on the outcome of variceal bleeding. Hepatology 2004;40:793801.

13. de Franchis R. Updating consensus in portal hypertension: report of the Baveno III consensus workshop on definitions, methodology and therapeutic strategies in portal hypertension. J Hepatol 2000;33: 846-52.

14. Abraldes JG, Gilabert R, Turnes J, et al. Utility of color Doppler ultrasonography predicting TIPS dysfunction. Am J Gastroenterol 2005;100:2696-701.

15. Villanueva C, Ortiz J, Sàbat M, et al. Somatostatin alone or combined with emergency sclerotherapy in the treatment of acute esophageal variceal bleeding: a prospective randomized trial. Hepatology 1999;30:384-9.

16. Bosch J, Thabut D, Bendtsen F, et al. Recombinant factor VIIa for upper gastrointestinal bleeding in patients with cirrhosis: a randomized, double-blind trial. Gastroenterology 2004;127:1123-30.

17. García-Pagán JC, Villanueva C, Albillos A, et al. Nadolol plus isosorbide mononitrate alone or associated with band ligation in the prevention of recurrent bleeding: a multicentre randomised controlled trial. Gut 2009;58:1144-50.

18. Dunnett CW, Gent M. An alternative to the use of two-sided tests in clinical trials. Stat Med 1996;15:1729-38.

19. Sackett DL. The principles behind the tactics of performing therapeutic trials. In: Haynes B, Sackett DL, Guyatt G, Tulliez M, eds. Clinical epidemiology. 3rd ed. Philadelphia: Lippincott Williams \& Wilkins, 2006:173-243.

20. Bureau C, García-Pagán JC, Otal P, et al. Improved clinical outcome using polytetrafluoroethylene-coated stents for TIPS: results of a randomized study. Gastroenterology 2004;126:469-75.

21. Bureau C, Pagan JC, Layrargues GP, et al. Patency of stents covered with polytetrafluoroethylene in patients treated by transjugular intrahepatic portosystemic shunts: long-term results of a randomized multicentre study. Liver Int 2007;27:742-7.

22. Bañares R, Casado M, Rodríguez-Láiz $J M$, et al. Urgent transjugular intrahepatic portosystemic shunt for control of acute variceal bleeding. Am J Gastroenterol 1998;93:75-9.

23. Malinchoc M, Kamath PS, Gordon FD, Peine CJ, Rank J, ter Borg PC. A model to predict poor survival in patients undergoing transjugular intrahepatic portosystemic shunts. Hepatology 2000;31: 864-71.

24. Papatheodoridis GV, Goulis J, Leandro G, Patch D, Burroughs AK. Transjugular intrahepatic portosystemic shunt compared with endoscopic treatment for prevention of variceal rebleeding: a metaanalysis. Hepatology 1999;30:612-22.

25. Luca A, D’Amico G, La Galla R, Midiri M, Morabito A, Pagliaro L. TIPS for prevention of recurrent bleeding in patients with cirrhosis: meta-analysis of randomized clinical trials. Radiology 1999;212: 411-21.

26. Escorsell A, Bañares R, García-Pagán JC, et al. TIPS versus drug therapy in preventing variceal rebleeding in advanced cirrhosis: a randomized controlled trial. Hepatology 2002;35:385-92.

Copyright (c) 2010 Massachusetts Medical Society.

RECEIVE IMMEDIATE NOTIFICATION WHEN

A JOURNAL ARTICLE IS RELEASED EARLY

To be notified when an article is released early on the Web and to receive the table of contents of the Journal by e-mail every Wednesday evening, sign up through our Web site at NEJM.org. 